\title{
Efeito dos ácidos naftaleno acético e indolilbutírico no enraizamento de estacas de jambolão [Syzygium cumini(L.) Skeels]
}

\author{
ALCANTARA, G.B.*; OLIVEIRA, Y.; LIMA, D.M.; FOGAÇA, L.A.; PINTO, F.; BIASI, L.A. \\ Departamento de Fitotecnia e Fitossanitarismo, Setor de Ciências Agrárias, Universidade Federal do Paraná \\ (UFPR), Rua dos Funcionários, 1540, Cabral, Caixa Postal 19061, CEP: 81531-990, Curitiba-Brasil \\ *giobomfim@hotmail.com
}

\begin{abstract}
RESUMO: O jambolão propaga-se normalmente por sementes o que acarreta variabilidade nas plantas descendentes e um problema quando o objetivo é a formação de pomar comercial. O desenvolvimento de protocolo de propagação vegetativa por meio da estaquia possibilitaria a reprodução de todas as características da planta matriz, uniformidade nas populações e facilidade de propagação. O presente trabalho teve por objetivo avaliar o efeito dos ácidos naftaleno acético (ANA) e indolilbutírico (AIB) no enraizamento de estacas de jambolão. Estacas da região mediana dos ramos foram confeccionadas com $12 \mathrm{~cm}$ de comprimento, cortadas em bisel na base e reto acima da última gema axilar, mantendo-se um par de folhas reduzidas à metade. As bases das estacas foram imersas por 10 segundos em soluções aquosas contendo ANA ou AIB nas concentrações de $0,500,1.000$ e $1.500 \mathrm{mg} \mathrm{L}^{-1}$. Para o plantio foram utilizadas bandejas plásticas contendo areia de granulometria média. As estacas foram mantidas em casa-de-vegetação com nebulização intermitente e após 120 dias do plantio, foram avaliadas as variáveis: porcentagem de estacas enraizadas, com calos, vivas (não enraizadas e sem calos) e mortas, comprimento das três maiores raízes $(\mathrm{cm})$ e número de raízes formadas por estaca. Os melhores resultados de enraizamento foram verificados com $1.000 \mathrm{mg} \mathrm{L}^{-1}$ para ambos os fitorreguladores testados. A porcentagem de enraizamento foi ligeiramente superior com a utilização de ANA quando comparada ao AIB.
\end{abstract}

Palavras-chave: plantas medicinais, estaquia, auxina, Syzygium cumine

\begin{abstract}
Effect of naphthaleneacetic acid and indolebutyric acid on rooting of jambul [Syzygium cumini (L.) Skeels] cuttings. Jambul usually propagates by seeds, which causes variability in the descendant plants and represents a problem in the formation of commercial orchards. The development of a protocol for vegetative propagation by cuttings would enable the reproduction of all features of the Mother plant, uniformity in populations and easy propagation. The aim of this work was to evaluate the effect of naphthaleneacetic acid (NAA) and indolebutyric acid (IBA) on rooting of jambul cuttings. Twelve-cm-long cuttings from the median region of branches were prepared through bevel cut in the base and right cut above the last axillary bud, keeping one pair of halved leaves. Cutting bases were immersed for 10 s in aqueous solutions containing NAA or IBA at 0, 500, 1000 and $1500 \mathrm{mg} \mathrm{L}^{-1}$ concentrations. Plastic trays containing medium sand were used in the planting. The cuttings were kept in a greenhouse under intermittent nebulization and, at 120 days after planting, the following variables were evaluated: percentage of rooted, with calluses, alive (not-rooted and without calluses) and dead cuttings; length of the three largest roots (cm); and number of roots per cutting. The best rooting was observed by using $1000 \mathrm{mg} \mathrm{L}^{-1}$ of both tested plant growth regulators. Rooting percentage was slightly higher under NAA relative to IBA.
\end{abstract}

Key words: medicinal plants, cutting, auxin, Syzygium cumine

\section{INTRODUÇÃO}

Pertencente à família Myrtaceae, o jambolão [Syzygium cumini (L.) Skeels] é espécie exótica originária da Índia, cultivada em vários países, inclusive no Brasil (Mazzanti et al., 2003). Esta espécie é conhecida na medicina popular indiana e paquistanesa por seus efeitos hipoglicemiantes

Recebido para publicação em 08/03/2009

Aceito para publicação em 30/03/2010

Rev. Bras. Pl. Med., Botucatu, v.12, n.3, p.317-321, 2010. 
(Prince et al., 1998). O jambolão é cultivado no Brasil como planta ornamental, no entanto, a casca, o fruto, a semente e a folha são frequentemente utilizados por pacientes diabéticos, sendo administrados na forma de diferentes preparados como extrato aquoso, extrato etanólico ou o suco da planta in natura (Mazzanti et al., 2003). O interesse medicinal do jambolão tem demandado informações a respeito das formas de propagação da espécie.

A propagação do jambolão, assim como a do jambeiro vermelho (Syzygium malaccense) e a do jambeiro rosa (Syzygium malacensis), ocorre por meio de sementes. Porém, esse é um método inviável, devido a problemas de segregação genética e ao longo período que as plantas levam para atingir a idade de produção (Gomes, 1984; Almeida et al., 2008). Poucos são os relatos sobre a produção de mudas de jambolão por estaquia, porém, estudos com jambeiro rosa (Syzygium malacensis) constataram que este pode ser propagado por meio de estacas com folhas apicais sem a utilização de ácido indolbutírico (AIB) (Martins et al., 2001).

A propagação vegetativa por estaquia é o método de propagação mais utilizado na produção comercial de diversas culturas ornamentais, medicinais e frutíferas tendo como vantagens a reprodução de todas as características da planta matriz, uniformidade nas populações e facilidade de propagação (Hartmann et al., 2002). Lima et al. (2007) avaliaram a influência do tipo de estaca e substrato no enraizamento de jambolão, e constataram que a maior porcentagem de enraizamento $(55 \%)$ e menor mortalidade (10\%) ocorreu em estacas retiradas da posição mediana do ramo mantidas em areia como substrato por 120 dias.

O uso de fitorreguladores tem sido utilizado como uma forma de aumentar o índice de enraizamento em espécies de difícil enraizamento. As auxinas são responsáveis por diversos efeitos fisiológicos na planta, dentre os quais se destaca a formação de raízes adventícias em estacas ocasionadas pelo alongamento e divisão celular. O ácido indolilacético (AIA) foi a primeira auxina descoberta e posteriormente outras substâncias sintéticas com atividade semelhantes ao AIA foram relatadas, dentre as quais alguns fitorreguladores utilizados na promoção da iniciação radicial, como o ácido indolil butírico (AIB) e o ácido naftaleno acético (ANA). As auxinas sintéticas são usualmente mais efetivas que o AIA por aparentemente não serem destruídas pelo sistema AIA-oxidase, persistindo nas plantas por um período de tempo maior que as auxinas endógenas. As auxinas conjugadas também são resistentes ao sistema AIA-oxidase (Salisbury \& Ross, 1992).

Para espécies de difícil enraizamento, a aplicação de fitorregulador pode compensar a falta do nível endógeno de auxina. O AIB e o ANA são as principais auxinas utilizadas no enraizamento de estacas, sendo que o AIB apresenta algumas vantagens quando comparado ao ANA, dentre as quais está a não toxicidade para uma maior amplitude de concentrações testadas, para a maioria das espécies vegetais (Hartmann et al., 2002).

Em estudo com duas espécies da família Myrtaceae, Cloezia buxifolia e Cloezia aquarum, foram comparadas as ações do AIB e do ANA, ambos aplicados em pó, no enraizamento de estacas. Para a C. buxifolia, tanto o AIB como o ANA, propiciaram aumento na porcentagem de enraizamento, no número e no comprimento de raízes. Porém estacas tratadas com AIB apresentaram melhores resultados para todas estas variáveis, chegando a atingir $75 \%$ de enraizamento, quando comparado ao ANA $(69,5 \%)$. Já para $C$. aquarum, o uso dos fitorreguladores teve efeito positivo na porcentagem de enraizamento, após 43 dias da instalação do experimento, no entanto, 40 dias depois essa tendência não foi verificada (Lemay et al., 2009). Na estaquia de jambeiro vermelho (Syzygium malaccense) e rosa (Syzygium malacensis), o AIB não apresentou efeito significativo no aumento da porcentagem de enraizamento, no número e no comprimento das raízes, quando comparado ao tratamento controle (Martins et al., 2001; Almeida et al., 2008).

O presente trabalho teve por objetivo avaliar o efeito dos ácidos naftaleno acético e indolilbutírico no enraizamento de estacas medianas de jambolão.

\section{MATERIAL E MÉTODO}

O experimento foi conduzido em casa-devegetação do Setor de Ciências Agrárias - UFPR, Curitiba, PR, entre os meses de maio e julho de 2007. As estacas foram retiradas de ramos semi-lenhosos de plantas matrizes de jambolão com seis anos de idade, da Estação Experimental do Canguiri, UFPR, localizada no município de Pinhais, PR.

As estacas medianas de cada ramo foram padronizadas com $12 \mathrm{~cm}$ de comprimento, cortadas em bisel na base e reto acima da última gema axilar, mantendo-se um par de folhas reduzidas à metade. Após o preparo, as estacas foram submetidas a tratamento fitossanitário com hipoclorito de sódio a $0,5 \%(\mathrm{v} / \mathrm{v})$ por 15 min e enxaguadas em água corrente durante $5 \mathrm{~min}$. As bases das estacas foram imersas por 10 segundos em soluções aquosas contendo ANA ou AIB em diferentes concentrações $(0,500,1.000$, 1.500 e $2.000 \mathrm{mg} \mathrm{L}^{-1}$ ). Para o plantio foram utilizadas bandejas plásticas perfuradas com dimensões de 42 x 28 x $10 \mathrm{~cm}$ contendo uma camada de $9 \mathrm{~cm}$ de areia de granulometria média como substrato.

As estacas foram mantidas em casa-devegetação com nebulização intermitente (das 8:00 às 17:00 $\mathrm{h}$ irrigação de $15 \mathrm{~s}$ a cada $15 \mathrm{~min}$; das 17:00

Rev. Bras. PI. Med., Botucatu, v.12, n.3, p.317-321, 2010. 
às 23:00 irrigação de $15 \mathrm{~s}$ a cada $1 \mathrm{~h}$ e das 23:00 às 8:00 h irrigação de $15 \mathrm{~s}$ a cada $3 \mathrm{~h}$ ) e após 120 dias do plantio, foram avaliadas a porcentagem de estacas enraizadas, com calos, vivas (que são as estacas que não enraizaram e também não formaram calos) e mortas, comprimento das três maiores raízes $(\mathrm{cm})$ e número de raízes formadas por estaca.

$O$ delineamento experimental utilizado foi o inteiramente casualizado, em esquema fatorial ( $2 \times 5)$, com 5 repetições e 16 estacas por unidade experimental. Para testar a homogeneidade das médias utilizou-se o teste de Bartlett e para a comparação de médias, os dados foram submetidos ao Teste de Tukey a $5 \%$ de probabilidade.

\section{RESULTADO E DISCUSSÃO}

Analisando a porcentagem de estacas enraizadas, verificou-se que $1.000 \mathrm{mg} \mathrm{L}^{-1}$ foi a melhor concentração para ambos os fitorreguladores testados (Figura 1). Concentrações de ANA e AIB acima de 1.000 $\mathrm{mg} \mathrm{L}^{-1}$ causam diminuição na porcentagem de enraizamento de estacas de jambolão (Figura 1). Concentrações excessivas de auxina podem inibir o desenvolvimento das raízes e brotações, causarem o amarelecimento e queda das folhas, necrose ou até ocasionarem a morte das estacas. Segundo Alvarenga \& Carvalho (1983), o aumento na concentração de auxinas, aplicadas em estacas, pode propiciar aumento no enraizamento até um ponto máximo, a partir do qual, qualquer acréscimo torna-se inibitório. Porém, quando concentrações de auxina são utilizadas abaixo do nível ideal, não há formação de raízes adventícias (Hartmann et al., 2002).

A concentração ótima para a aplicação do fitorregulador, que irá estimular o crescimento e diferenciação dos tecidos, causando aumento na porcentagem de enraizamento, irá depender do nível
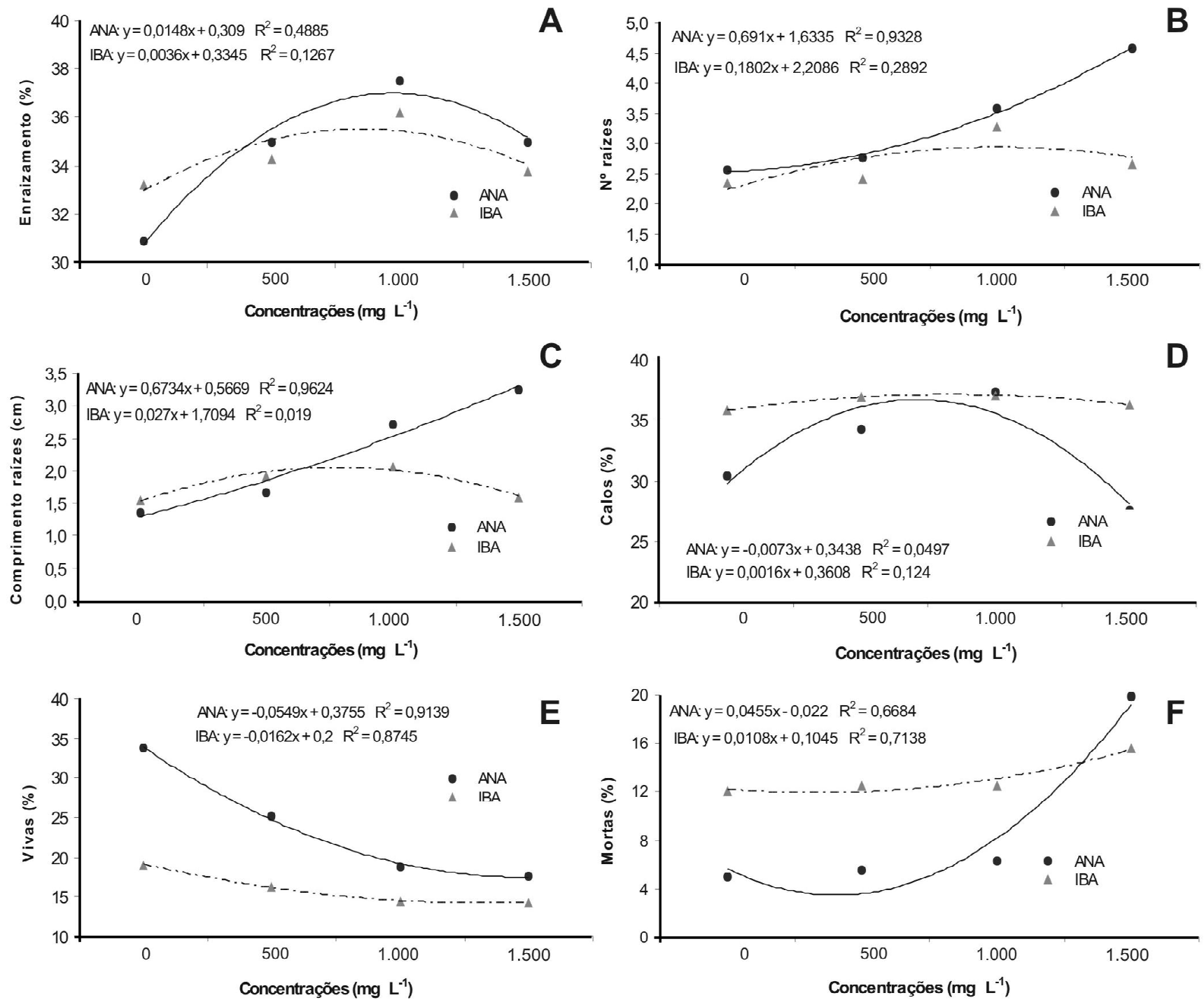

FIGURA 1. Porcentagens de enraizamento $(A)$, número de raízes por estaca $(B)$, comprimento médio das três maiores raízes $(C)$, formação de calos $(D)$, sobrevivência $(E)$ e mortalidade $(F)$ de estacas de jambolão (nome científico) em diferentes concentrações $\left(0,500,1.000\right.$ e $\left.1.500 \mathrm{mg} \mathrm{L}^{-1}\right)$ de ANA e AIB.

Rev. Bras. PI. Med., Botucatu, v.12, n.3, p.317-321, 2010. 
endógeno do hormônio, combinado com outros promotores do enraizamento (Hartmann et al., 2002). Em estudo com pessegueiro [Prunus persica (L.) Batch] verificou-se que a maior porcentagem de enraizamento (56\%) ocorreu com a aplicação de 250 $\mathrm{mg} \mathrm{L}^{-1}$ de AIB e que concentrações de 1.000 a 2.500 $\mathrm{mg} \mathrm{L}^{-1}$ inibiram a formação de raízes, por apresentarem efeito fitotóxico às estacas (Tworkoski \& Takeda, 2007).

Lima et al. (2007) constataram porcentagem de enraizamento maior (55\%) que as do presente trabalho, utilizando o mesmo tipo de estaca e sem o uso de fitorregulador. Isto se deve possivelmente ao fato de que as estacas utilizadas por Lima et al. (2007) terem sido coletadas de plantas matrizes mais jovens, cinco anos, que as do presente trabalho, seis anos. A idade da planta matriz da qual são retiradas às estacas pode influenciar no enraizamento, pois estacas coletadas de plantas matrizes mais velhas são mais difíceis de enraizar quando comparadas com estacas provenientes de plantas juvenis (Greenwood et al., 1991). Mudanças nas características morfológicas, fisiológicas e bioquímicas que ocorrem em diversas partes da planta têm sido relacionadas com a variação da idade da planta (Hartmann et al., 2002).

Com a utilização do ANA foi verificada porcentagem de enraizamento ligeiramente superior ao AIB, em praticamente todas as concentrações testadas. Biasi et al. (2002) verificaram que a utilização de AIB não apresentou efeito positivo na emissão de raízes em estacas semilenhosas de caquizeiro 'Fuyu' (Diospyros kakı), demonstrando que, apesar de ser a auxina sintética mais utilizada, pode não ocasionar resultados satisfatórios em algumas espécies. Wendling (2002) verificou com clones de Eucalyptus grandis que a aplicação de AIB não resultou em aumento no enraizamento e sobrevivência das miniestacas, sendo observada influência negativa das doses acima de 500 $\mathrm{mg} \mathrm{L}^{-1}$.

Diferentemente do observado no presente trabalho, Husen \& Pal (2007) testaram duas concentrações de ANA e AIB (2.000 e $4.000 \mathrm{mg} \mathrm{L}^{-1}$ ) em estacas coletadas de brotações medianas de Teca (Tectona grandis) e verificaram melhor resposta ao AIB quando comparado ao ANA, com porcentagens de 65,5 e $73,5 \%$ com $2.000 \mathrm{mg} \mathrm{L}^{-1}$ ANA e AIB, respectivamente e, 60,3 e 75,2\% com $4.000 \mathrm{mg} \mathrm{L}^{-1}$ ANAe AIB, respectivamente.

As variáveis número de raízes formadas por estaca e comprimento das três maiores raízes foram influenciados de forma positiva com o aumento da concentração de ANA, sendo que as melhores respostas foram observadas com $1500 \mathrm{mg} \mathrm{L}^{-1}$ (Figura 1). No entanto, com essa concentração foi verificado menor porcentagem de enraizamento, quando comparada a $1000 \mathrm{mg} \mathrm{L}^{-1}$. O regulador de crescimento pode acelerar o metabolismo normal e aumentar 0 número de primórdios radiculares, mesmo que não aumente a porcentagem de enraizamento. Fato este que já havia sido relatado por Hartmann et al. (2002), que declara que a aplicação de auxina pode proporcionar maior velocidade de formação, qualidade e uniformidade do sistema radicial.

Com o uso de ambos reguladores (ANA e AIB) foi verificada maior formação de calos a 1.000 $\mathrm{mg} \mathrm{L}^{-1}$. Com ANA houve diminuição de calos com concentrações maiores. Já com o AIB a formação de calos foi constante, com as diferentes concentrações testadas (Figura 1). Todas as concentrações de ambos os reguladores tenderam a favorecer a redução da porcentagem de estacas vivas, que não enraizaram e não chegaram a formar calos. A não utilização de regulador, assim como as menores concentrações de reguladores testadas (500 e $1000 \mathrm{mg} \mathrm{L}^{-1}$ ) diminuiram a porcentagem de estacas mortas.

Nas condições do presente trabalho concluise que os tratamentos com $1.000 \mathrm{mg} \mathrm{L}^{-1}$ de ANA e AIB foram mais efetivos no enraizamento de estacas de jambolão. O tratamento com ANA proporcionou maior porcentagem de enraizamento. $O$ aumento das concentrações de ANA até a concentração de 1.500 $\mathrm{mg} \mathrm{L}^{-1}$ possibilitou a formação de maior número e comprimento das raízes.

\section{REFERÊNCIA}

ALMEIDA, E.J. et al. Propagação de jambeiro vermelho (Syzygium malaccense L.) por estaquia de ramos herbáceos. Bioscience Journal, v.24, n.1, p.39-45, 2008. ALVARENGA, L.R.; CARVALHO, V.D. Uso de substâncias promotoras de enraizamento de estacas de frutíferas. Informe Agropecuário, v.9, n.101, p.47-55, 1983.

BIASI, L.A. et al. Potencial organogenético de tecidos caulinares e radiculares de caquizeiro. Revista Brasileira de Fruticultura, v.24, n.1, p.29-34, 2002.

GOMES, R.P. Fruticultura Brasileira. 8.ed. São Paulo: Nobel, 1984. 446p.

GREENWOOD, M.S.; FOSTER, G.S.; AMERSON, H.V. Vegetative propagation of southern pines. In: DURYEA, M.L.; DOUGHERTY, P.M. (Eds.). Forest regeneration manual. Dordrecht: Kluwer Academic, 1991. p.75-86. HARTMANN, H.T. et al. Plant propagation: principles e practices. 7.ed. New Jersey: Prentice Hall, 2002. 880p. HUSEN, A.; PAL, M. Effect of branch position and auxin treatment on clonal propagation of Tectona grandis Linn. f. New Forests, v.34, n.3, p.223-33, 2007.

LEMAY, V.; GÂTEBLÉ, G.; McCOY, S. Vegetative propagation of two endemic species of Cloezia Brongn. \& Gris for conservation and mining revegetation activities in New Caledonis. New Forests, v. 37, n.1, p.1-8, 2009.

LIMA, Y.O.U. et al. Tipos de estacas e substratos no enraizamento de jambolão. Scientia Agraria, v.8, n.4, p.44953, 2007.

MARTINS, A.B.G.; GRACIANO, F.A.; SILVA, A.V.C. Clonagem de Jambeiro-rosa (Syzygium malacensis) por estaquia de 
ramos enfolhados. Revista Brasileira de Fruticultura, v.23, n.2, p.365-8, 2001.

MAZZANTI, C.M. et al. Extrato da casca de Syzygium cumini no controle da glicemia e estresse oxidativo de ratos normais e diabéticos. Ciência Rural, v.33, n.6, p.1061-5, 2003.

PRINCE, P.S.M.; MENON, V.P.; PARI, L. Effect of Syzygium cumini extracts on hepatic herxokinase and glucose-6phosphatase en experimental diabetes. Phytotherapy Research, v.11, n.7, p.529-31, 1998.
SALISBURY, F.B.; ROSS, C.W. Plant Physiology. 4.ed. Califórnia: Wadsworth, 1992. 682p.

TWORKOSKI, T.; TAKEDA, F. Rooting response of shoot cuttings from three peach growth habits. Science Horticulture, v.115, n.1, p.98-100, 2007.

WENDLING, I. Rejuvenescimento de clones de Eucalyptus grandis por miniestaquia seriada e micropropagação. 2002. 98p. Tese (Doutorado - Área de concentração em Ciência Florestal) - Departamento de Ciência Florestal, Universidade Federal de Viçosa, Viçosa. 